

LAW RENCE LIVERMORE N A T IO N A L LABORATORY

\title{
Nondipole Effects in Xe 4d Photoemission
}

O. Hemmers, R. Guillemin, A. Wolska, D. W. Lindle, D. Rolles, K. T. Cheng, W. R. Johnson, H. L. Zhou, S. T. Manson

July 14,2004

The 14th International Conference on Vacuum Ultraviolet Radiation Physics

Cairns, Australia July 19, 2004 through July 23, 2004 
This document was prepared as an account of work sponsored by an agency of the United States Government. Neither the United States Government nor the University of California nor any of their employees, makes any warranty, express or implied, or assumes any legal liability or responsibility for the accuracy, completeness, or usefulness of any information, apparatus, product, or process disclosed, or represents that its use would not infringe privately owned rights. Reference herein to any specific commercial product, process, or service by trade name, trademark, manufacturer, or otherwise, does not necessarily constitute or imply its endorsement, recommendation, or favoring by the United States Government or the University of California. The views and opinions of authors expressed herein do not necessarily state or reflect those of the United States Government or the University of California, and shall not be used for advertising or product endorsement purposes. 


\title{
Nondipole effects in Xe $4 d$ photoemission
}

\author{
O. Hemmers ${ }^{a}$ R. Guillemin ${ }^{\mathrm{a}, \mathrm{b}}$ A. Wolska ${ }^{\mathrm{a}, \mathrm{b}}$ D. W. Lindle ${ }^{\mathrm{a}}$ \\ D. Rolles ${ }^{\mathrm{b}}$ K. T. Cheng ${ }^{\mathrm{c}}$ W. R. Johnson ${ }^{\mathrm{d}}$ H. L. Zhou ${ }^{\mathrm{e}}$ \\ S. T. Manson ${ }^{\mathrm{e}}$ \\ ${ }^{a}$ Department of Chemistry, University of Nevada, Las Vegas, NV 89154-4003 \\ ${ }^{\mathrm{b}}$ Advanced Light Source, Lawrence Berkeley National Laboratory, Berkeley, \\ California 94720 \\ ${ }^{\mathrm{c}}$ University of California, Lawrence Livermore National Laboratory, Livermore, \\ California 94550 \\ ${ }^{\mathrm{d}}$ Department of Physics, University of Notre Dame, Notre Dame, Indiana 46556 \\ ${ }^{\mathrm{e}}$ Department of Physics and Astronomy, Georgia State University, Atlanta, GA \\ 30303-3083
}

\begin{abstract}
We measured the nondipole parameters for the spin-orbit doublets $\mathrm{Xe} 4 d_{5 / 2}$ and $\mathrm{Xe} 4 d_{3 / 2}$ over a photon-energy range from $100 \mathrm{eV}$ to $250 \mathrm{eV}$ at beamline 8.0.1.3 of the Advanced Light Source at the Lawrence Berkeley National Laboratory. Significant nondipole effects are found at relatively low energies as a result of Cooper minima in dipole channels and interchannel coupling in quadrupole channels. Most importantly, sharp disagreement between experiment and theory, when otherwise excellent agreement was expected, has provided the first evidence of satellite twoelectron quadrupole photoionization transitions, along with their crucial importance for a quantitatively accurate theory.
\end{abstract}

Key words: Photoionization, Atoms, Nondipolar Electron Angular Distributions, Valence Shells

PACS: 31.25.Eb, 32.80.Fb

Over the past decade or so there has been an upsurge in both experimental and theoretical studies of nondipole effects in atomic and molecular photoionization [1] owing to advances in experimental capabilities, notably thirdgeneration synchrotron light sources. These studies have revealed significant nondipole effects not only at multi-keV photon energies but at hundreds and even tens of eV.

Email address: hemmers@unlv.nevada.edu (O. Hemmers). 


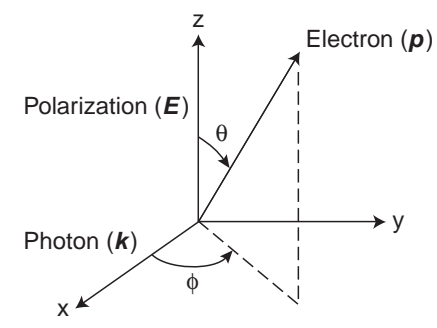

Fig. 1. Geometry applicable to photoelectron angular-distribution measurements using polarized light.

In addition to the intrinsic interest in photoelectron angular distributions, studies of nondipole angular-distribution effects provide information on the relatively weak ionizing quadrupole transitions, both their amplitudes and their phases, information which is otherwise inaccessible. Of particular interest here is quadrupole transitions in photoionization connecting the initial discrete state of the photoionization process to final continuum states of different angular momentum and parity from those connected by dipole transitions, thereby facilitating study of the quadrupole-allowed continua.

Measurements over the 100-250 eV photon energy range were made at the Advanced Light Source (ALS) of the Lawrence Berkeley National Laboratory on undulator beamline 8.0.1 during several 2-bunch periods. This mode is crucial for time-of-flight (TOF) spectroscopy. The spherical grating monochromator (SGM) houses three interchangeable gratings with the lowest energy of $78 \mathrm{eV}$ at $1.9 \mathrm{GeV}$ ring energy. The experimental chamber consists of five electron TOF-analyzers in a CoNetic shielded chamber for gas-phase measurements, which is described in [2]. Ne $2 s, \operatorname{Ar} 2 p$, and He $1 s$ photolines were used to calibrate the analyzer transmissions because the dipole and nondipole contributions to their angular distributions are now well known. The degree of linear polarization of the synchrotron light was determined to be $>99.9 \%$. Electron analyzers were positioned at sets of angles that are sensitive to different combinations of the dipole parameter, $\beta$, and the nondipole parameters $\delta$, and $\gamma$. Linear combinations of the measured photoelectron intensities yielded values of $\gamma+3 \delta=\zeta$.

Calculations were carried out using relativistic random-phase approximation (RRPA) methodology [3,4]. RRPA includes significant aspects of ground-state correlation, along with interchannel coupling among all of the photoionization channels that are included. In the present work, all relativistic single ionization and excitation channels from the $4 s, 4 p_{1 / 2}, 4 p_{3 / 2}, 4 d_{3 / 2}, 4 d_{5 / 2}, 5 s, 5 p_{1 / 2}$, and $5 p_{3 / 2}$ subshells of Xe are considered, a total of 20 interacting dipole and 25 interacting quadrupole channels. This calculation is entirely ab initio except experimental binding energies have been used. This methodology has been 


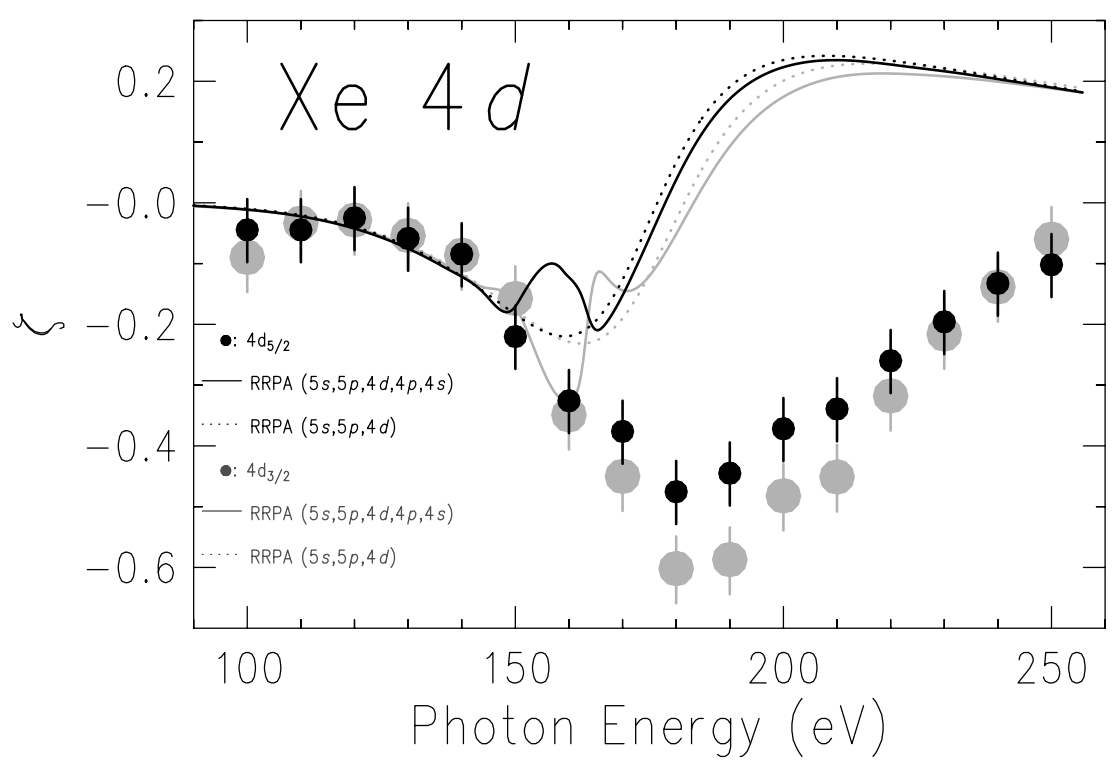

Fig. 2. Nondipole angular distribution parameter $\zeta$ for the $\mathrm{Xe} 4 d_{5 / 2}$ and $4 d_{3 / 2}$ photoelectrons in comparison with RRPA calculations with interchannel coupling amongst different channels.

found to give excellent results for Xe $4 d$ dipole photoionization [5] and Xe $5 s$ nondipole photoionization [6], both in the same energy range considered herein.

The experimental results for the nondipole of $\zeta$ parameter are shown in Fig. 2 as a function of photoelectron energy. A notable feature of the measurement is the $4 d_{3 / 2}$ nondipole parameter $\zeta$ which reaches a value of about -0.6 , and the $4 d_{5 / 2}$ nondipole parameter $\zeta$ which reaches a value of about -0.5 , at a photoelectron energy of about $110 \mathrm{eV}$, which corresponds to a photon energy of about $180 \mathrm{eV}$, the region just above the $4 p$ thresholds. Also shown in Fig. 2 are the results of our RRPA calculations. Below a photoelectron energy of about $80 \mathrm{eV}$, the theoretical result is in excellent agreement with experiment. At higher energies, from about $80 \mathrm{eV}$ to $180 \mathrm{eV}$, starting around the $4 p$ ionization thresholds, agreement is poor; the experiment shows a broad region in which the nondipole parameter $\zeta$ takes on large negative values, then slowly increases with increasing energy. The theoretical result, on the other hand, shows significant differences in the behaviors of the $4 d_{3 / 2}$ and $4 d_{5 / 2}$ channels only in the immediate neighborhood of the $4 p$ thresholds, followed by a rapid rise to small positive values of $\zeta$ and a slightly decreasing plateau region. This disagreement is quite surprising in view of the excellent agreement found for the nondipole parameter in the case of Xe $5 s$ photoionization in the same energy region with the same theoretical formulation [6]. We note that the next order corrections, including the octupole channels, have been calculated and found to be negligible. 
Measurements of spin-orbit resolved nondipole parameters for the Xe $4 d$ spinorbit doublet has been performed, and dynamical effects of the spin-orbit interaction are seen. Significant nondipole effects are found at relatively low energy as a result of Cooper minima in dipole channels and interchannel coupling in quadrupole channels. Most importantly, sharp disagreement between experiment and theory, when otherwise excellent agreement was expected, has provided the first evidence of satellite two-electron quadrupole photoionizing transitions, along with their crucial importance for a quantitatively accurate theory. Our results point to the need of a theoretical method, which simultaneously treats discrete-state correlation in initial and final-ionic states, interchannel coupling amongst the various ionization channels, relativistic interactions, both dipole and quadrupole transitions, and inner shells; all of which are required for a quantitative understanding of the Xe $4 d$ nondipole parameters in this energy region.

The UNLV group acknowledges support by NSF Grant No. PHY-01-40375. DR acknowledges the financial support through the ALS Doctoral Fellowship in Residence program. The work of KTC was performed under the auspices of DOE by the University of California, LLNL under Contract No. W-7405ENG-48. The research of WRJ was supported in part by NSF Grant No. PHY01-39928. The research of HLZ was supported by NSF and NASA. The work of STM was supported by DOE, Division of Chemical Sciences, BES grant No. DE-FG02-03ER15428. The ALS (LBNL) was supported by DOE, Materials Science Division, BES, OER under Contract No. DE-AC03-76SF00098.

\section{References}

[1] O. Hemmers, R. Guillemin, and D.W. Lindle, Radiat. Phys. Chem. 70, 123 (2004) and references therein.

[2] O. Hemmers, S. B. Whitfield, P. Glans, H. Wang, D. W. Lindle, R. Wehlitz, and I. A. Sellin, Rev. Sci. Instrum. 69, 3809 (1998).

[3] W.R. Johnson and C.D. Lin, Phys. Rev. A 20, 964 (1979).

[4] W.R. Johnson, C.D. Lin, K.T. Cheng, and C.M. Lee, Phys. Scr. 21, 409 (1980).

[5] H. Wang, G. Snell, O. Hemmers, M.M. Sant'Anna, I.A. Sellin, N. Berrah, D.W. Lindle, P.C. Deshmukh, N. Haque, and S.T. Manson, Phys. Rev. Lett. 87, 123004 (2001).

[6] O. Hemmers, R. Guillemin, E.P. Kanter, B. Krässig, D.W. Lindle, S.H. Southworth, R. Wehlitz, J. Baker, A. Hudson, M. Lotrakul, D. Rolles, W.C. Stolte, I.C. Tran, A. Wolska, S.W. Yu, M.Ya. Amusia, K.T. Cheng, L.V. Chernysheva, W.R. Johnson, and S.T. Manson, Phys. Rev. Lett. 91, 053002 (2003). 